\title{
Optics Elements for Modeling Electrostatic Lenses and Accelerator Components: III. Electrostatic Deflectors
}

T.A. Brown and G.H. Gillespie

This article was submitted to $8^{\text {th }}$ International Conference on Accelerator Mass Spectrometry Vienna, Austria

U.S. Department of Energy September 6-10, 1999

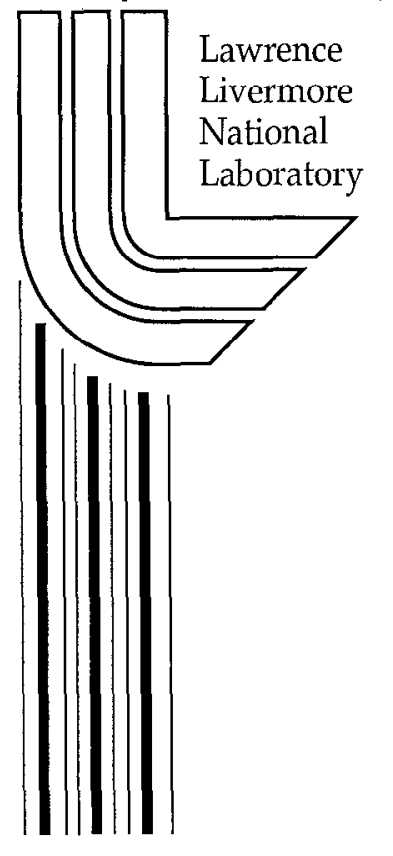

\section{September 21, 1999}




\section{DISCLAIMER}

This document was prepared as an account of work sponsored by an agency of the United States Government. Neither the United States Government nor the University of California nor any of their employees, makes any warranty, express or implied, or assumes any legal liability or responsibility for the accuracy, completeness, or usefulness of any information, apparatus, product, or process disclosed, or represents that its use would not infringe privately owned rights. Reference herein to any specific commercial product, process, or service by trade name, trademark, manufacturer, or otherwise, does not necessarily constitute or imply its endorsement, recommendation, or favoring by the United States Government or the University of California. The views and opinions of authors expressed herein do not necessarily state or reflect those of the United States Government or the University of California, and shall not be used for advertising or product endorsement purposes.

This is a preprint of a paper intended for publication in a journal or proceedings. Since changes may be made before publication, this preprint is made available with the understanding that it will not be cited or reproduced without the permission of the author.

This report has been reproduced directly from the best available copy.

Available to DOE and DOE contractors from the

Office of Scientific and Technical Information

P.O. Box 62, Oak Ridge, TN 37831

Prices available from (423) 576-8401

http://apollo.osti.gov/bridge/

Available to the public from the National Technical Information Service

U.S. Department of Commerce 5285 Port Royal Rd., Springfield, VA 22161 http://www.ntis.gov/

\section{OR}

Lawrence Livermore National Laboratory Technical Information Department's Digital Library http://www.llnl.gov/tid/Library.html 


\begin{abstract}
Manuscript for submission to the Journal
Nuclear Instrument and Methods in Physics Research B

as a contribution to the

Proceeding of the Eighth International Conference on Accelerator Mass Spectrometry Vienna, Austria, September 6-10, 1999.
\end{abstract}

Authors:

T. A. Brown

Center for Accelerator Mass Spectrometry, LLNL, P.O. Box 808, Livermore, CA 94551, U.S.A.

and

G. H. Gillespie

G.H. Gillespie Associates, Inc., P.O. Box 2961, Del Mar, CA 92014, U.S.A.

Title:

Optics Elements for Modeling Electrostatic Lenses and Accelerator Components: III. Electrostatic Deflectors 


\title{
Optics Elements for Modeling Electrostatic Lenses and Accelerator Components: III. Electrostatic Deflectors
}

\author{
T. A. Brown \\ Center for Accelerator Mass Spectrometry, LLNL, P.O. Box 808, Livermore, CA 94551, U.S.A. \\ and \\ G. H. Gillespie \\ G.H. Gillespie Associates, Inc., P.O. Box 2961, Del Mar, CA 92014, U.S.A.
}

\begin{abstract}
Ion-beam optics models for simulating electrostatic prisms (deflectors) of different geometries have been developed for the computer code TRACE 3-D. TRACE 3-D is an envelope (matrix) code, which includes a linear space charge model, that was originally developed to model bunched beams in magnetic transport systems and radiofrequency (RF) accelerators. Several new optical models for a number of electrostatic lenses and accelerator columns have been developed recently that allow the code to be used for modeling beamlines and accelerators with electrostatic components. The new models include a number of options for: (1) Einzel lenses, (2) accelerator columns, (3) electrostatic prisms, and (4) electrostatic quadrupoles. A prescription for setting up the initial beam appropriate to modeling 2-D (continuous) beams has also been developed. The models for electrostatic prisms are described in this paper. The electrostatic prism model options allow the modeling of cylindrical, spherical, and toroidal electrostatic deflectors. The application of these models in the development of ionbeam transport systems is illustrated through the modeling of a spherical electrostatic analyzer as a component of the new low energy beamline at CAMS.
\end{abstract}

Keywords: Mass spectrometry; Computer codes; Electrostatic optics; Beam deflectors. Corresponding Author:

T. A. Brown, CAMS L-397, LLNL, P.O. Box 808, Livermore, CA 94551, U.S.A fax: 925-423-7884, telephone: 925-423-8507, email: tabrown@1lnl.gov 


\section{Introduction}

The Center for Accelerator Mass Spectrometry (CAMS) at the Lawrence Livermore National Laboratory [1] has implemented a variety of improvements to its HVEC Model FN Tandem Electrostatic Accelerator based AMS system in the last several years. Several software tools have been used to support the design of the new beamlines and components, and to carry out performance studies for the improvements [e.g., 2,3]. One of the new tools used for that work is an enhanced version of the TRACE 3-D program that includes several electrostatic ionbeam optics elements. Einzel lens and electrostatic acceleration column models, which have been described previously [4,5], were used for simulating previous beamline and low energy acceleration sections of the AMS system. As a further enhancement, an electrostatic deflector (prism) model has also been developed, and it is described here. The spherical electrostatic analyzer (ESA) option of this model was used in the development of the new low energy ESAsector/Magnetic-sector beamline at CAMS, and the utilization of the model in this developmental work is discussed below.

\section{Electrostatic Deflector Model for TRACE 3-D}

The TRACE 3-D program [6] is an envelope code that includes a first order space charge model. The code is a standard program used in the design of radiofrequency (RF) linacs and transport systems for high-current bunched beams. In previous papers, we outlined the use of the program for simulating high-current continuous (DC) beams, and described the development of additional optical elements that model Einzel lenses [4], DC accelerator columns and gaps [5], and electrostatic quadrupoles [7]. The previous studies presented the derivation of the optical models from a first principles approach starting with the electric fields of the elements. For the electrostatic deflectors discussed here, we rely on the detailed treatment provided by Wollnik [8], relating the first order transfer matrices implemented for TRACE 3-D to the work of Wollnik.

TRACE 3-D simulates the ion-beam envelope evolution through a series of optical elements for the full six-dimensional phase space of the beam. Space charge impulses are applied at steps 
along the beamline whose maximum separation distances are specified by the user. Each optical element in the program is defined by a transfer matrix for each step. Many of the transfer matrices are identical to the linear (first-order) R-matrices used in particle optics calculations [9]. However, TRACE 3-D numerically integrates the envelope equations step-by-step, so that other effects such as fringe fields, longitudinal field variations, and certain nonlinearities can be readily incorporated into the element descriptions. The approach is sometimes referred to as a differential R-matrix model, where effective (not necessarily linear) R-matrix elements are computed at each step. The simulation describes the evolution of the second moments of the beam distribution function. For the electrostatic deflector, we have defined differential Rmatrices based on results from Wollnik [8]. While only the linear, uniform field terms are included in the present description; the model could be extended in a manner analogous to that of other work [4-7].

The electrostatic deflector is assumed to have mid-plane symmetry and the defection plane is taken to be the $\mathrm{x}-\mathrm{z}$ plane. The geometry for first-order optics can be defined in terms of two radii [8]: $r_{e o}$, the radius of curvature of a particle (reference particle) moving on the equipotential surface between the two deflector plates corresponding to the beam initial voltage, and $R_{e o}$, the inner electrode radius. The geometry is illustrated in Figure 1.

(Figure 1. Here)

The $6 \times 6 \mathrm{R}$-matrix for static systems with mid-plane symmetry reduces to the following general form (see equation 2.30 of reference [9]):

$$
R=\left[\begin{array}{cccccc}
R_{11} & R_{12} & 0 & 0 & 0 & R_{16} \\
R_{21} & R_{22} & 0 & 0 & 0 & R_{26} \\
0 & 0 & R_{33} & R_{34} & 0 & 0 \\
0 & 0 & R_{43} & R_{44} & 0 & 0 \\
R_{51} & R_{52} & 0 & 0 & 1 & R_{56} \\
0 & 0 & 0 & 0 & 0 & 1
\end{array}\right]
$$


The non-trivial elements of the R-matrix can be expressed in terms of the step size $\Delta z / 2$, the reference trajectory curvature $h=1 / r_{e o}$, the reference trajectory's relativistic energy variable $\gamma$, and two dimensionless parameters $p$ and $q$ :

$$
\begin{aligned}
& \mathrm{R}_{11}=\mathrm{R}_{22}=\cos [\mathrm{ph}(\Delta z / 2)] \\
& \mathrm{R}_{12}=\sin [\mathrm{ph}(\Delta z / 2)] /(\mathrm{ph}) \\
& \mathrm{R}_{21}=-\mathrm{ph} \sin [\mathrm{ph}(\Delta z / 2)] \\
& \mathrm{R}_{33}=\mathrm{R}_{44}=\cos [\mathrm{ph}(\Delta z / 2)] \\
& \mathrm{R}_{34}=\sin [\mathrm{qh}(\Delta z / 2)] /(\mathrm{qh}), \\
& \mathrm{R}_{43}=-\mathrm{qh} \sin [\mathrm{qh}(\Delta z / 2)] \\
& \mathrm{R}_{16}=-\mathrm{R}_{52}=\{1-\cos [\mathrm{ph}(\Delta z / 2)]\} /\left(\mathrm{p}^{2} \mathrm{~h}\right) \\
& \left.\mathrm{R}_{26}=-\mathrm{R}_{51}=\sin [\mathrm{ph}(\Delta z / 2)]\right\} / \mathrm{p}, \\
& \text { and } \quad \mathrm{R}_{56}=\{\mathrm{ph}(\Delta z / 2)-\sin [\mathrm{ph}(\Delta z / 2)]\} /(\mathrm{ph})+(\Delta z / 2) / \gamma^{2}
\end{aligned}
$$

The type of deflector under consideration determines the dimensionless parameters $\mathrm{p}$ and $\mathrm{q}$. In general, $p$ and $q$ depend upon the reference trajectory's relativistic velocity variable $\beta$, and the ratio of the reference trajectory radius to the radius of curvature of the inner electrode, $r_{e o} / R_{e o}$. The values of $\mathrm{p}$ and $\mathrm{q}$ for cylindrical $\left(r_{e o} / R_{e o}=0\right)$, toroidal $\left(0<r_{e o} / R_{e o}<1\right)$ and spherical $\left(r_{e d} / R_{e o}=1\right)$ electrostatic prisms are summarized in Table 1.

(Table 1. Here)

The 6-D phase space envelopes are calculated for each step size of $\Delta z / 2$ by applying the Rmatrix of Equations (1) - (10) to the transformation of the $6 \times 6$ beam $\sigma$-matrix [6]. A spacecharge impulse matrix is applied between each $\Delta z / 2$ step to model the linear space-charge forces, for either bunched [6] or continuous [7] beams. The $x, y$ and $z$ beam envelopes at each location $z$ are given by the square roots of the three sigma matrix elements $\sigma_{11}, \sigma_{33}$ and $\sigma_{55}$ at $z$. The eight R-matrix elements given by Equations (2)-(7) determine the transverse focusing properties of the deflector, while the two matrix elements $R_{16}$ and $R_{26}$ describe the dispersion effects. The three matrix elements $R_{51}, R_{52}$ and $R_{56}$ determine the longitudinal effects of the 
deflector, which can be important when modeling bunched beams. For the continuous beams considered in the next section, $R_{51}, R_{52}$ and $R_{56}$ have negligible impact.

\section{Application to the new CAMS Low-energy Beamline}

The spherical analyzer option of the electrostatic deflector model described above, together with the three-tube Einzel lens and two-tube acceleration gap models described previously [4,5], were used in the development of the new ESA-sector/Magnetic-sector ion-injection beamline that has been installed as the second low energy AMS beamline attached to our "Dual $90^{\circ}$ " injection magnet at CAMS. The ion-beam transport through the new beamline, as simulated by the enhanced version of TRACE 3-D (PowerTrace ${ }^{\mathrm{TM}}$ ), is shown in Figure 2.

(Figure 2. Here)

The first elements of the beamline are two Einzel lenses (positions 3 and 6) arranged as a zoom lens that allows control of both the position of, and the ion-beam divergence at, the following beam waist. Based on a series of beamline models (and other considerations including available physical space), a $750-\mathrm{mm}$ central-radius-of-curvature $\left(r_{e o}\right) 90^{\circ}$ spherical electrostatic analyzer was chosen for the new beamline. The ESA was designed to operate at up to $10 \mathrm{kV}$ on each electrode $(+/-)$ to meet the desired condition that the beamline be able to transport ions with energies up to $\sim 65 \mathrm{keV}$ energy. The ESA was designed with a $100 \mathrm{~mm}$ gap between electrodes and the electrodes are $240 \mathrm{~mm}$ tall.

The modeled ESA (position 10) acts as expected as a 1R-1R double-focusing beamline element. The ESA image position falls near the first of two 2-tube acceleration columns (positions 13 and 21) that have been used to simulate the insulating gaps that electrically isolate the magnet vacuum chamber from the upstream and downstream beamline components. In typical operation for ${ }^{13} \mathrm{C}^{-}$and ${ }^{14} \mathrm{C}^{-}$, the potential of the vacuum chamber is switched in less than $100 \mu$ s between 2 potentials (nominally $-0.3 \mathrm{kV}$ for $30 \mathrm{~ms}$ and $-2 \mathrm{kV}$ for $300 \mathrm{~ms}$ ). These potentials are chosen so that the deceleration in the first gap and subsequent acceleration in the 
second gap allows the "Dual $90^{\circ "}$ magnetic-sector selected mass to be alternated between short pulses of the ${ }^{13} \mathrm{C}$ ions and longer pulses of the ${ }^{14} \mathrm{C}$ radioisotope. The "Dual $90^{\circ}$ " magnet image falls near the second of the 2-tube acceleration columns and the final Einzel lens (position 24) is used to match the ion-beam diameter to the acceptance of the low energy accelerator tubes.

In initial tests of the installed ESA, it was found that the ESA did not behave as a $1 \mathrm{R}-1 \mathrm{R}$ double-focusing element as expected for a spherical electrostatic deflector, or as modeled by the first-order PowerTrace spherical electrostatic deflector model. Rather, the ESA had a shorter horizontal focal length and longer vertical focal length. Subsequent modeling with PowerTrace and Laplace-equation-based electric field programs showed that the ESA was behaving as a toroidal electrostatic analyzer with a vertical radius of curvature $\left(R_{e o}\right)$ greater than $800 \mathrm{~mm}$, and led to the conclusion that the physical design of the ESA was the cause of the "toroidal" behavior.

As was discussed above, the differential R-matrix model of the electrostatic deflector is based on the first-order transfer matrices presented by Wollnik [8]. Hence, the model results are accurate to the degree that the physical characteristics of the modeled deflector produce electrostatic fields that are consistent with the first-order analysis. In the case of the installed ESA, it is clear that the physical design did not produce electric fields within the ESA that were consistent with those expected for a symmetrical-potential spherical electrostatic analyzer. As was mentioned above, the methods used in developing the PowerTrace Einzel lens and acceleration column models could be used to extend the electrostatic deflector model to allow more accurate modeling of actual physical geometries.

In the case of the installed ESA, detailed SIMION modeling was undertaken within CAMS, with the goals of understanding the design factors which contributed to the "non-spherical" behavior of the ESA, and of guiding efforts to improve the ESA's performance. This modeling effort was based on a mm-resolution 3-D grid representation of the actual physical dimensions of the installed ESA. This modeling effort provided a solution under which the ESA now functions properly as a double-focusing element. 


\section{Summary}

We have developed a differential R-matrix model that calculates beam-envelope evolution during the transport of an ion-beam through electrostatic deflectors. This electrostatic deflectors model allows the simulation of spherical, cylindrical, and toroidal deflectors. The differential Rmatrix model is based on the first-order analysis presented by Wollnik (1), and hence, the model results are accurate to the degree that the physical characteristics of the modeled deflector produce electrostatic fields that are consistent with the first-order analysis. The electrostatic deflector model is a part of a suite of enhancements to the electrostatic capabilities of TRACE 3D. The other components of the electrostatic suite allow modeling of Einzel lenses, electrostatic quadrupoles, and acceleration columns and gaps. This suite of electrostatic models, together with the normal elements provided by TRACE 3-D, have allowed the modeling of the AMS system at the Center for Accelerator Mass Spectrometry. In particular, the spherical electrostatic analyzer option of the deflectors model was used in the development of the recently installed low-energy $90^{\circ}$ ESA-sector $/ 90^{\circ}$ Magnetic-sector beamline that is now operational and being used in a variety of AMS studies.

\section{Acknowledgements}

This work was performed in part under the auspices of the U.S. Department of Energy at the Lawrence Livermore National Laboratory under contract W-7405-Eng-48. PowerTrace ${ }^{\mathrm{TM}}$ is available from AccelSoft Inc., www.ghga.com/accelsoft.

\section{References}

[1] M.L. Roberts, G.S. Bench, T.A. Brown, M.W. Caffee, R.C. Finkel, S.P.H.T. Freeman, L.J. Hainsworth, M. Kashgarian, J.E. McAninch, I.D. Proctor, J.R. Southon, and J.S. Vogel, Nucl. Instr. And Methods B 123 (1997) 57. 
[2] T.J. Ognibene, T.A. Brown, J.P. Knezovich, M.L. Roberts, J.R. Southon and J.S. Vogel, "Ion-optics calculations of the LLNL compact AMS system for biochemical ${ }^{14} \mathrm{C}$ measurements," these proceedings (1999).

[3] T.A. Brown, "Ion-source modeling and improved performance of the CAMS high-intensity Cs-sputter ion source," these proceedings (1999).

[4] G.H. Gillespie and T.A. Brown, Proc. 1997 IEEE Particle Accel. Conf., (1998) 2559.

[5] G.H. Gillespie and T.A. Brown, Nucl. Instr. And Methods A 427 (1999) 315.

[6] K. Crandall and D. Rusthoi, "TRACE 3-D Documentation," 3rd ed., Los Alamos National Laboratory Report LA-UR-97-886 (1997) 106 pp.

[7] G.H. Gillespie, Proc. XIX International LINAC Conf., ANL-98/28 (1998) 150.

[8] H. Wollnik, in: Focusing of Charged Particles, ed. A. Septier (Academic Press, New York, 1967).

[9] D.C. Carey. The Optics of Charged Particle Beams. (Harwood Academic Publishers, New York, 1987). 


\section{Figure Captions}

Figure 1. The deflector electrode and ion trajectory geometry used for the electrostatic deflector model. The radius of curvature of a reference particle moving on the equipotential surface between the two deflector plates corresponding to the beam initial potential is indicated by $r_{e o}$. The inner electrode radius is indicated by $R_{e o}$. For a toroidal deflector $R_{e o} \neq r_{e o}$, and for a cylindrical deflector $R_{e o}=\infty$.

Figure 2. PowerTrace output for the new $90^{\circ}$-ESA-sector $/ 90^{\circ}$-Magnetic-sector ion-injection beamline that has recently been installed as the second low energy AMS beamline at CAMS. The Einzel lenses at 3 and 6 have been adjusted to transport the ion beam $\left(80 \mathrm{~mA}{ }^{12} \mathrm{C}\right)$ cleanly through the vertical gap of the injection magnet at 17. The Einzel lens at 24 has been adjusted to match the beam envelope to the acceptance of the accelerator beam tubes. The spherical electrostatic deflector at 10 has been modeled as a $90^{\circ}$ bend with a $750 \mathrm{~mm}$ central radius of curvature, $r_{e o}$. 


\section{Tables}

Table 1. The relativistic formulations of the parameters $\mathrm{p}$ and $\mathrm{q}$ for cylindrical, toroidal, and spherical electrostatic deflectors (after [8]).

\begin{tabular}{cccc}
\hline Parameter & cylindrical & toroidal & spherical \\
$\mathrm{p}$ & $\left(2-\beta^{2}\right)^{1 / 2}$ & $\left(2-\left[r_{e o} / R_{e o}\right]-\beta^{2}\right)^{1 / 2}$ & $\left(1-\beta^{2}\right)^{1 / 2}$ \\
$\mathrm{q}$ & 0 & {$\left[r_{e o} / R_{e o}\right]^{1 / 2}$} & 1 \\
\hline
\end{tabular}




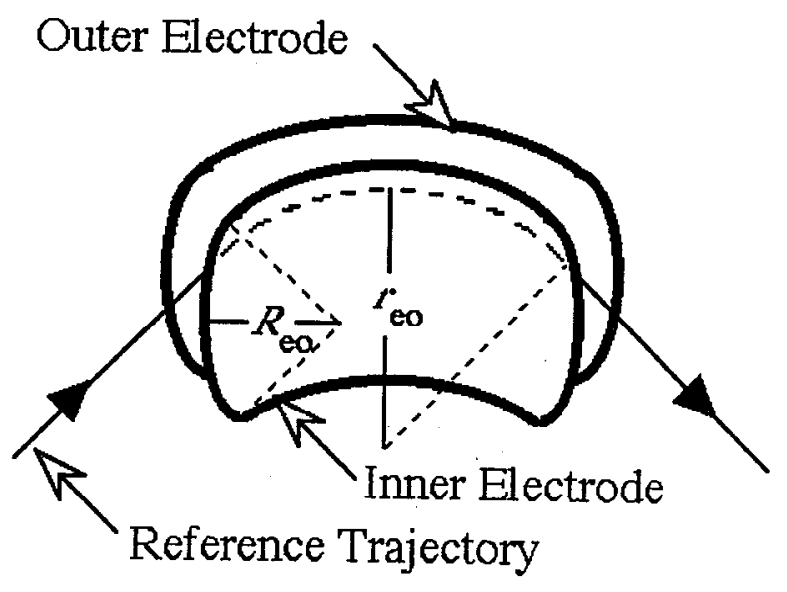




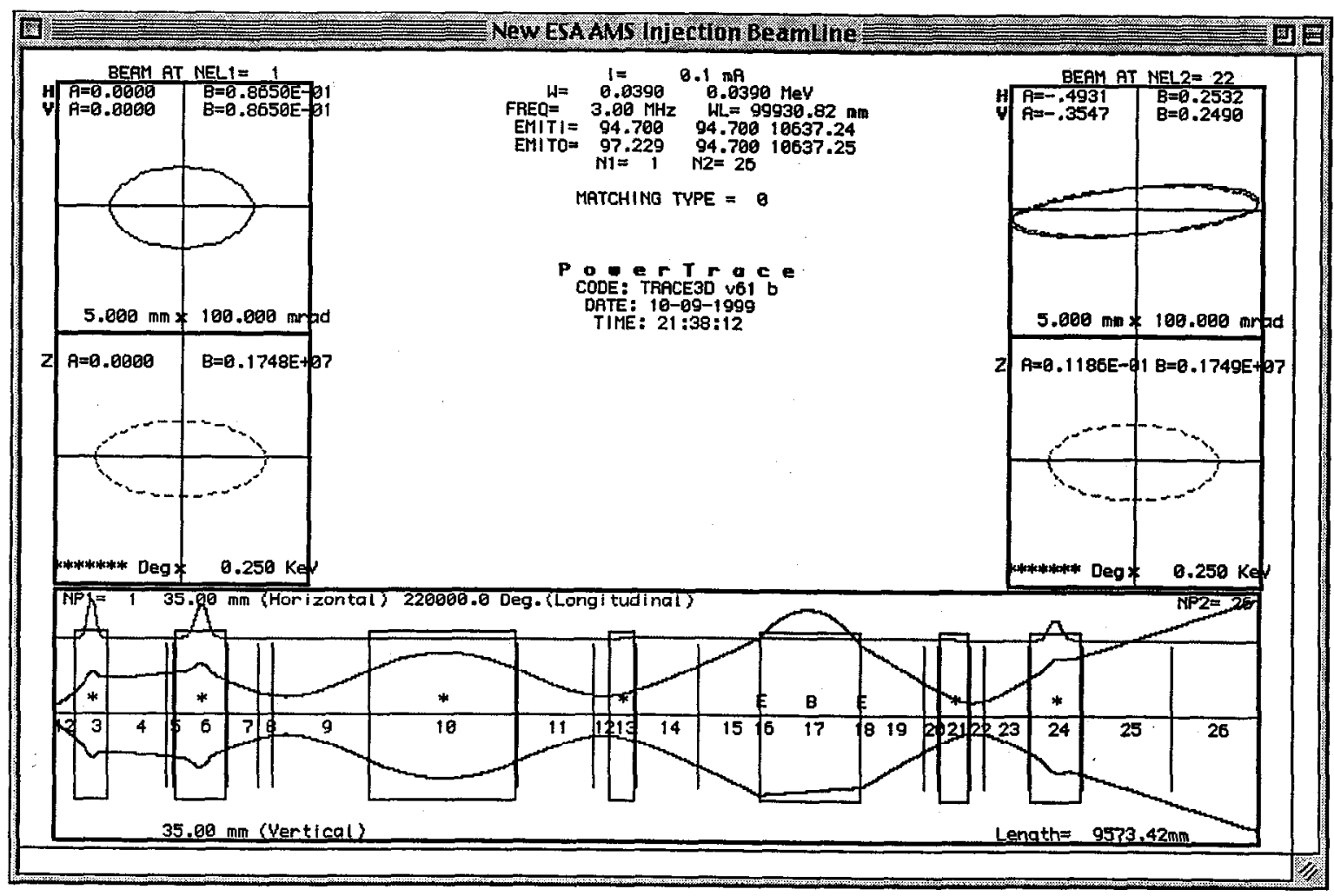

